Chronic Obstructive Pulmonary Diseases: Journal of the COPD Foundation

\title{
Journal Club: COPD and Rehospitalization
}

\author{
Ron Balkissoon, MD, MSc, DIH, FRCPC ${ }^{1}$
}

Abbreviations: chronic obstructive pulmonary disease, COPD; Centers for Medicare and Medicaid Services, CMS; Roflumilast and Inhaled Corticosteroid/Long-Acting 32 -Agonist on Chronic Obstructive Pulmonary Disease Exacerbations, RE(2)SPOND; inhaled corticosteroid/long-acting beta-agonists, ICS/LABA; long-acting muscarinic antagonists, LAMA; phosphodiesterase-4, PDE4; Roflumilast and Exacerbations in Patients receiving Appropriate Combination Therapy, REACT

Citation: Balkissoon R. Journal Club: COPD and Rehospitalization. Chronic Obstr Pulm Dis (Miami). 2016;3(4):791-797. doi: http:// dx.doi.org/10.15326/jcopdf.3.4.2016.0167

1 Denver, Colorado

\section{Address correspondence to:}

Ron Balkissoon, MD, MSc, DIH, FRCPC

balkissoonr@njhealth.org

\section{Keywords:}

chronic obstructive pulmonary disease; COPD; rehospitalization

\section{COPD and Rehospitalization}

Chronic obstructive pulmonary disease (COPD) exacerbations may account for up to $75 \%$ of direct costs associated with care for these patients. ${ }^{1}$ Hospitalization of COPD patients accounts for a substantial part of that economic burden. It is estimated that medical costs attributable to COPD in the United States may reach $\$ 49$ billion by $2020 .^{2}$ As the paper by Roberts and colleagues, (included below), points out, $20 \%$ of Medicare patients hospitalized for COPD have an all cause rehospitalization within 30 days of discharge and $30 \%$ of these readmissions are for COPD-related events. In 2012, the Centers for Medicare and Medicaid Services (CMS) implemented the Hospital Readmissions Reduction Program, that penalizes hospitals that have excessive hospital readmissions within 30 days by reducing their Medicare payments. In 2014 this program was expanded to include COPDrelated exacerbation readmissions. Unfortunately while these penalties are being implemented there is a dearth of evidence-based guidance as to strategies that are likely to reduce early rehospitalization. (See also the 2nd National Readmissions Summit Report in this issue.) In this Journal Club we focus on studies that are examining factors that may predict and or influence the rates of COPD-related rehospitalization.
The first article by Roberts et al is a retrospective analysis of data from a national U.S. health claims data base and identifies factors that predict increased risk of rehospitalization in addition to early ( $<30$ days) versus late ( $>30$ days) rehospitalization. The 2 following articles look at the potential efficacy of roflumilast in reducing exacerbations and/or rehospitalization. The "Roflumilast and Inhaled Corticosteroid/Long-Acting $\beta 2$-Agonist on Chronic Obstructive Pulmonary Disease Exacerbations (RE(2)SPOND)" study has been eagerly anticipated as the counterpart to the European study "Roflumilast and Exacerbations in Patients receiving Appropriate Combination Therapy (REACT)"3 to look at the benefit of roflumilast added to inhaled corticosteroid/longacting beta-agonists (ICS/LABA) and/or long-acting muscarinic antagonists (LAMA). The study suggests that a phenotype of patients that have a history of frequent hospitalization may predict individuals likely to benefit from treatment with roflumilast. The second study presents interesting data regarding the relationships between rehospitalization rates and early versus late institution of roflumilast therapy post hospital discharge. Finally, the last 2 articles examine the efficacy of 2 different approaches to health coaching programs and reducing early rehospitalization rates. It is likely that the economic burden related to COPD will continue to grow for at least the next few decades and it is prudent to initiate efforts to try to control costs related to the treatment of this patient population. Recognition of the complexity of this COPD cohort in terms of different phenotypes and their multiple comorbidities is critical to developing approaches that improve health care quality while also reducing costs. 


\section{Abstract 1 \\ A retrospective analysis to identify predictors of COPD-related rehospitalization}

Roberts MH, Clerisme-Beaty E, Kozma CM, Paris A, Slaton T, Mapel DW. BMC Pulm Med. 2016; 16(1):68. doi: http://dx.doi.org/10.1186/s12890-016-0231-3

\section{BACKGROUND:}

Chronic obstructive pulmonary disease (COPD) is often associated with recurrent hospitalizations. This study aimed to identify factors related to COPD rehospitalization.

\section{METHODS:}

A national US claims database was used to identify patients, aged $\geq 40$ years, hospitalized for COPD. Their first COPD-related hospital admission date in 2009 was set as the index date, with post-discharge COPD-related rehospitalization assessed for 180 days post-index date. Data were analyzed for: 1) all eligible patients in whom early COPD-related rehospitalization was evaluated (1-30 days post discharge; all-patient cohort) and 2) a patient subset not rehospitalized early in whom late COPD-related rehospitalization was evaluated ( $>30$ days post discharge to 180 days post-index date; late cohort). Logistic regressions controlling for age and sex assessed potential COPDrelated rehospitalization predictors. Variables from the 360-day pre-index period and index hospitalization were evaluated for each cohort, and 30-day postdischarge variables evaluated for the late cohort.

\section{RESULTS:}

Of 3612 patients with an index hospitalization, $4.8 \%$ (174) had an early COPD-related rehospitalization, and of the remaining 3438 patients, $13.7 \%$ (471) had a late COPD-related rehospitalization. Several pre-index variables were predictive of early COPDrelated rehospitalization including: pneumonia; comorbidities; COPD-related drug therapies; and prior hospitalizations. In patients not rehospitalized early, the strongest predictor of late COPD-related rehospitalization was pre-index COPD-related hospitalization $(\mathrm{OR}=3.64[\mathrm{P}<0.001])$. The strongest index hospitalization factors predictive of late COPDrelated rehospitalization were use of steroids (any route: $\mathrm{OR}=1.62[\mathrm{P}=0.007])$ and nebulizers $(\mathrm{OR}=1.65$
$[\mathrm{P}=0.007])$; neither predicted early COPD-related rehospitalization. Generally, factors predicting COPDrelated rehospitalization were similar in both cohorts.

\section{CONCLUSIONS:}

Several pre-index variables were associated with COPD-related rehospitalization. A strong predictor of COPD-related rehospitalization was prior hospitalization during the pre-index period, particularly with a primary COPD diagnosis, whilst other predictive factors related to increased COPD severity; these may be useful indicators for COPDrelated rehospitalization risk assessment. Some factors, e.g., recurrent pneumonia and exacerbations, may be modifiable.

\section{KEYWORDS:}

Chronic obstructive pulmonary disease (COPD); Comorbidity; Exacerbations; Readmission predictors; Rehospitalization; Utilization

\section{Comments}

This retrospective analysis had a relatively unique data source that contained data from inpatient pharmacies, as well as medical and outpatient pharmacies. Perhaps it is no surprise that prior hospitalization in the previous year and pneumonia were predictive of early rehospitalization. Other comorbidities that were associated with early rehospitalization included anxiety, asthma, diabetes, hypoxia, ischemic heart disease and dyspnea while notably, hypertension, pulmonary vascular disease, stroke, depression and congestive heart failure were not. Late rehospitalization seemed to be associated with the same comorbidities as early rehospitalization in addition to congestive heart failure, depression, hypertension and osteoporosis and pulmonary vascular disease. Drug-related therapies looked at antibiotics, inhaled steroids, non-inhaled steroids, long-acting beta-agonists and short-acting beta-agonists and anticholinergics. Interestingly pre-index inhaled steroid use was not predictive of an early rehospitalization but was predictive of a late COPD-related hospitalization. All the other classes of medications were predictive of both late and early hospitalizations. One interpretation of this data could be that inhaled steroid use may be protective against early rehospitalization. Interestingly, pre-index influenza or pneumonia vaccines were also predictive of early COPD hospitalization. The findings 
from this study can provide useful information in the design of prospective studies with regard to parameters that should be included for developing risk stratification systems and specific interventions that will reduce the rate of COPD rehospitalizations.

\section{Abstract 2 Effect of roflumilast and inhaled corticosteroid/long-acting $\beta 2$-agonist on chronic obstructive pulmonary disease exacerbations (RE(2) SPOND). A randomized clinical trial}

Martinez FJ, Rabe KF, Sethi S, et al. Am J Respir Crit Care Med. 2016; 194(5):559-567.

doi: http://dx.doi.org/10.1164/rccm.201607-13490C

\section{RATIONALE:}

Moderate and severe exacerbations are incompletely prevented by maximal inhalation therapy in patients with severe chronic obstructive pulmonary disease.

\section{OBJECTIVES:}

To determine whether roflumilast reduces moderate and/or severe chronic obstructive pulmonary disease exacerbations in patients at risk for exacerbations despite treatment with inhaled corticosteroid/longacting $\beta 2$-agonist with or without a long-acting muscarinic antagonist (LAMA).

\section{METHODS:}

In this 52-week, phase 4, double-blind, placebocontrolled RE(2)SPOND (Roflumilast Effect on Exacerbations in Patients on Dual [LABA/ICS] Therapy) trial (NCT01443845), participants aged 40 years or older with severe/very severe chronic obstructive pulmonary disease, chronic bronchitis, two or more exacerbations and/or hospitalizations in the previous year, and receiving inhaled corticosteroid/ long-acting $\beta 2$-agonist with or without LAMA daily for 3 or more months were equally randomized to once-daily roflumilast, $500 \mu \mathrm{g}(\mathrm{n}=1,178)$, or placebo $(n=1,176)$. Stratification was based on LAMA use.

\section{MEASUREMENTS AND MAIN RESULTS:}

Although rate of moderate or severe exacerbations per patient per year (primary endpoint) was reduced by $8.5 \%$ with roflumilast versus placebo, the between- group difference was not statistically significant (rate ratio, 0.92; 95\% confidence interval, 0.811.04; $\mathrm{P}=0.163$ ). However, roflumilast improved lung function, and in a post hoc analysis roflumilast significantly reduced the rate of moderate or severe exacerbations in participants with a history of more than three exacerbations and/or one or more hospitalizations in the prior year. Adverse eventrelated discontinuations occurred in $11.7 \%$ roflumilasttreated and $5.4 \%$ placebo-treated participants. Deaths occurred in $2.5 \%$ roflumilast and $2.1 \%$ placebo participants.

\section{CONCLUSIONS:}

Roflumilast failed to statistically significantly reduce moderate and/or severe exacerbations in the overall population. Roflumilast improved lung function and reduced exacerbations in participants with frequent exacerbations and/or hospitalization history. The safety profile of roflumilast was consistent with that of previous studies. Clinical trial registered with www. clinicaltrials.gov (NCT01443845).

\section{KEYWORDS:}

bronchodilators; clinical trial; hospitalization; phosphodiesterase-4 inhibitor

PMID: 27585384

\section{Comments}

Roflumilast is a phosphodiesterase-4 (PDE4) inhibitor that had been shown in earlier trials to reduce the risk of exacerbations in patients with COPD not on other maintenance medications reported to reduce exacerbations such as ICSs/LABAs and/or LAMAs. The trial Roflumilast and Exacerbations in Patients Receiving Appropriate Combination Therapy (REACT) trial was a European study published last year that used the enteric coated version of roflumilast and demonstrated the efficacy of roflumilast in combination with ICS/LABA with or without LAMA in reducing moderate to severe exacerbations in patients with 2 or more exacerbations and/or hospitalizations in the prior 12 months. The Food and Drug Administration-approved formulation of roflumilast is not film (enteric) coated and thus required a separate study of efficacy with the similar study design. The authors for the RESPOND trial provide a very thoughtful discussion of possible factors that may contribute to why the REACT trial met the primary endpoint (reduction in moderate to severe exacerbations) 
whereas the RESPOND trial did not. The investigators point to the fact that RESPOND had 10\% more patients with very severe airflow obstruction and a higher proportion of patients with more than 2 exacerbations (24\%-25\%) versus in the REACT trial (10\%-11\%). There were also differences in the definitions of exacerbations and definitions of "end of exacerbations". Further, the highest dose of ICS/LABA allowed in the REACT was fluticasone/salmeterol 500/50 whereas in RESPOND only the 250/50 dose was allowed. The RESPOND trial did not initially allow patients to be on LAMA but later amended the protocol to allow LAMA use, and this may explain the lower percentage of patients that were on triple therapy (ICS/LABA/LAMA) in the RESPOND trial. The difference in the formulations with regard to film coating was felt to not likely contribute to the difference as studies have shown bioequivalence between the formulations and there were no differences between the studies in bioequivalence. Two subgroups (men and those on fluticasone/salmeterol 250/50) did show statistically significant reductions in rates of moderate and severe exacerbations for roflumilast versus placebo in the RESPOND study. About $65 \%$ of participants in both treatment arms were on fluticasone/ salmeterol and 35\% on budesonide/formoterol. Also, the authors report that there was a lower number of patients with 3 or more prior exacerbations in the RESPOND study compared to the REACT study. Hence, there are several study design issues that may provide plausible explanations and insights as to why there were differences in results for RESPOND versus REACT. While the RESPOND trial did not meet its primary endpoint for a broad population of COPD patients it perhaps helped to further refine our definition of a subphenotype (i.e., previous history of hospitalization and/ or frequent [4 or more] exacerbations in the previous 12 months) that are likely to experience a significant reduction in moderate to severe exacerbations.

\section{Abstract 3 Benefits of early roflumilast treatment after hospital or emergency department discharge for a COPD exacerbation}

Lee Q, Mocarski M, Sun SX. Am Health Drug Benefits. 2016; 9(3):140-150

\section{BACKGROUND:}

Chronic lower respiratory disease, which includes chronic obstructive pulmonary disease (COPD), is the third leading cause of death in the United States. Roflumilast is an oral, once-daily, selective phosphodiesterase-4 inhibitor approved for reducing the risk for COPD exacerbations in patients with severe COPD associated with chronic bronchitis and a history of exacerbations.

\section{OBJECTIVES:}

To evaluate the effects of roflumilast treatment timing on COPD exacerbation rates (primary objective) and on resource utilization and healthcare costs (secondary objective) after hospital or emergency department discharge associated with a COPD exacerbation.

\section{METHODS:}

In this retrospective cohort study, claims data from March 2011 to March 2013 were extracted from Truven Health MarketScan combined commercial healthcare claims and Medicare supplemental claims databases and were analyzed to compare the exacerbation rates and the healthcare resource utilization and costs between the early roflumilast treatment (treatment initiation $\leq 30$ days after hospital or emergency department discharge) and the delayed roflumilast treatment (treatment initiation 31-180 days after discharge) cohorts. Multivariate logistic regression and generalized linear models with loglink function and gamma distribution were adjusted for age, sex, insurance plan type, COPD disease complexity, and comorbidities.

\section{RESULTS:}

A total of 995 patients ( $N=280$ early roflumilast treatment, $\mathrm{N}=715$ delayed roflumilast treatment) were included. Compared with the delayed roflumilast treatment group, patients in the early roflumilast treatment group were $39 \%$ less likely to have an exacerbation after hospital discharge $(P=.004)$. The patients receiving early roflumilast treatment also had $42 \%(P=.003)$ and $37 \%(P=.005)$ lower risks for COPD-related and all-cause rehospitalizations, respectively, than patients in the delayed roflumilast treatment group. Significantly fewer patients receiving early roflumilast treatment had moderate $(P=.013)$ or severe $(P=.002)$ exacerbations. Early roflumilast treatment also was associated with reduced annualized COPD-related $(\mathrm{P}=.012)$ and allcause $(P=.009)$ rehospitalizations, outpatient visits per patient ( $\mathrm{P}<.001$ for COPD-related and all-cause), and procedures or therapies (COPD-related, $\mathrm{P}=.016$; all-cause, $\mathrm{P}=.009)$. The early treatment group had 
fewer COPD-related emergency department visits per patient than the delayed roflumilast treatment group $(P=.035)$, and the total mean annualized COPDrelated and all-cause costs were reduced by $\$ 7273(\mathrm{P}=$ .014 ) and $\$ 14,111$ ( $\mathrm{P}=.002)$, respectively. Multivariate analyses showed that early treatment was associated with lower COPD-related and all-cause annualized health services costs per patient annually $(\mathrm{P}<.001$ for both).

\section{CONCLUSION:}

In this real-world study, the patients with COPD who initiated roflumilast treatment $\leq 30$ days after a hospital or emergency department discharge for a COPD-related exacerbation experienced fewer subsequent exacerbations and rehospitalizations, reduced healthcare utilizations, and lower healthcare costs than the patients who delayed their roflumilast treatment.

\section{KEYWORDS:}

chronic obstructive pulmonary disease; exacerbations; healthcare costs; healthcare utilization; hospital discharge; rehospitalization; roflumilast

PMID: 27606039

\section{Comments}

This is a retrospective data base analysis that compares early versus late initiation of roflumilast therapy post discharge and suggests that early institution of therapies in the first 30 days post discharge was associated with a reduction in early readmissions. Patients with asthma were not excluded from this analysis and this clearly influences how one should evaluate the data. While one must be mindful of the limitations of a retrospective study like this with regard to such factors as misdiagnosis between asthma and COPD and that patients were not randomly and evenly assigned to early versus late institution of roflumilast, it certainly provides enough food for thought as to the merits of examining this question in a prospective study.

\section{Abstract 4 Health coaching and COPD rehospitalization: A randomized study}

Benzo R, Vickers K, Novotny PJ, et al. Am J Respir Crit Care Med. 2016 Mar 8. [Epub ahead of print]

\section{RATIONALE:}

Hospital readmission in Chronic Obstructive
Pulmonary Disease (COPD) has attracted attention due to the burden to patients and the Health Care system. There is a knowledge gap on approaches to reduce $C O P D$ readmissions.

\section{OBJECTIVE:}

Determine the effect of comprehensive Health Coaching, on the rate of COPD readmissions.

\section{METHODS:}

215 hospitalized for a COPD exacerbation were randomized at hospital discharge to Motivational Interviewing based Health Coaching plus a written action plan for exacerbations and a brief exercise advice versus usual care. Measures Rate of COPD related hospitalization during the year-followup.

\section{RESULTS:}

The Absolute Risk Reduction of COPD related rehospitalization by health coaching was $7.5 \% \mathrm{p}=0.01$, $11.0 \% \quad \mathrm{p}=0.02, \quad 11.6 \% \mathrm{p}=0.03, \quad 11.4 \% \mathrm{p}=0.05$ and $5.4 \% \mathrm{p}=0.24$ at $1,3,6,9$, and 12 months compared to the control group. The Odds Ratio for COPD Hospitalization on the Intervention Arm Compared to the Control was 0.08 (CI 0.01-0.70) at 1 month post discharge, 0.35 (CI 0.13-0.93) at 3 months post discharge, 0.30 (CI $0.12-0.73$ ) at 6 months and 0.49 (CI 012-1.11) at 1 year post discharge. Missing value rate for the primary outcome was $0.4 \%$ ( 1 patient). Disease specific quality of life improved significantly in the health coaching group compared to the control group at 6 and 12 months by the Chronic Respiratory Questionnaire (CRQ) Emotional Score (EmotionMastery) and Physical Score (Dyspnea-Fatigue) $\mathrm{p}<0.05$. There were no differences in the physical activity measured at any time points between groups. Conclusion Health Coaching may represent a feasible and possibly effective intervention to reduce COPD readmissions. Clinical trial registration available at www.clinicaltrials.gov, ID NCT01058486.

\section{KEYWORDS:}

COPD; Motivational Interviewing; Quality of Life; health coaching; self-management PMID: 26953637

\section{Comments}

This study points out the value of early follow up, discharge education and patient empowerment as being very effective in reducing hospital readmissions. 
In particular, it did show a significant impact on 30 day readmission rate as well as readmissions at 3 months and 6 months. It is also relatively low cost. It is interesting to note that it is reported that there was no difference in the rate of use of antibiotics and steroids between the 2 groups suggesting that it wasn't simply the factor of having easier access to medications or greater use of these medications in the intervention group. It would have been interesting to have more information on this particular aspect of the study. It is not explicitly stated that the control group were also given an action plan with a package containing 5 days of prednisone and antibiotics. This is encouraging data presenting a relatively affordable intervention that does seem to reduce early rehospitalization.

\section{Abstract 5 The E-coach technology-assisted care transition system: a pragmatic randomized trial}

Ritchie CS, Houston TK, Richman JS, et al. Transl Behav Med. 2016;6(3):428-437

Care transitions from the hospital to home remain a vulnerable time for many patients, especially for those with heart failure (CHF) and chronic obstructive pulmonary disease (COPD). Despite regular use in chronic disease management, it remains unclear how technology can best support patients during their transition from the hospital. We sought to evaluate the impact of a technology-supported care transition support program on hospitalizations, days out of the community and mortality. Using a pragmatic randomized trial, we enrolled patients (511 enrolled, 478 analyzed) hospitalized with CHF/COPD to "E-Coach," an intervention with condition-specific customization and in-hospital and post-discharge support by a care transition nurse (CTN), interactive voice response post-discharge calls, and CTN followup versus usual post-discharge care (UC). The primary outcome was 30-day rehospitalization. Secondary outcomes included (1) rehospitalization and death and (2) days in the hospital and out of the community. E-Coach and UC groups were similar at baseline except for gender imbalance $(p=0.02)$. After adjustment for gender, our primary outcome, 30-day rehospitalization rates did not differ between the E-Coach and UC groups (15.0 vs. $16.3 \%$, adjusted hazard ratio [95\% confidence interval]: 0.94 [0.60, 1.49]). However, in the COPD subgroup, E-Coach was associated with significantly fewer days in the hospital (0.5 vs. 1.6, $\mathrm{p}=0.03$ ). E-Coach, an IVR-augmented care transition intervention did not reduce rehospitalization. The positive impact on our secondary outcome (days in hospital) among COPD patients, but not in CHF, may suggest that E-Coach may be more beneficial among patients with COPD.NIH trial registry number: NCT01135381Trial Protocol: http://dx.doi.org/ 10.1016/j.cct.2012.08.007.

\section{KEYWORDS:}

Care transitions; Self-management; Telehealth PMID: 27339715 PMCID: PMC4987612

\section{Comments}

I added this recently published study that looked at an interactive telephonic system that included motivational coaching before discharge but then was followed with an interactive voice response system that would ask set questions and based on responses might prompt preset educational responses for the patient or send red flag alerts that would lead to a nurse contacting the patient. They did not receive any package of medications to self administer at early signs of an exacerbation. This was a study of both cardiac patients and COPD patients and was negative for the primary outcome of rehospitalization within 30 days, though the COPD group did have few days in hospital overall. These 2 studies may point to some of the limitations of E-coaching versus live coaching systems and/or the impact of having an emergency package of medications to take home and start at the earliest signs of an exacerbation. 


\section{References}

1. American Thoracic Society/European Respiratory Society. Standards for the diagnosis and management of patients with COPD. Version 1.2. American Thoracic Society website. www. thoracic.org/copd-guidelines. Published 2004. Accessed April 30, 2008.

2. Ford ES, Murphy LB, Khavjou O, Giles WH, Holt JB, Croft JB. Total and state-specific medical and absenteeism costs of COPD among adults aged $\geq 18$ years in the United States for 2010 and projections through 2020. Chest. 2015; 147(1): 31-35. doi: http:// dx.doi.org/10.1378/chest.14-0972

3. Martinez FJ, Calverley PMA, Goehring U-M, Brose M, Fabbri LM, Rabe KF. Effect of roflumilast on exacerbations in patients with severe chronic obstructive pulmonary disease uncontrolled by combination therapy (REACT): a multicentre randomised controlled trial. Lancet. 2015; 385(9971): 857-866. 